\title{
A multivalent PDZ-domain protein assembles signalling complexes in a G-protein-coupled cascade
}

\author{
Susan Tsunoda, Jimena Sierralta, Yumei Sun, Ruth Bodner, Emiko Suzuki, Ann Becker, Michael Socolich \\ \& Charles S. Zuker
}

Howard Hughes Medical Institute and Departments of Biology and Neurosciences, University of California at San Diego, La Jolla, California, 92093-0649, USA * The Insitute of Medical Science, The University of Tokyo, Japan

How are signalling molecules organized into different pathways within the same cell? In Drosophila, the inaD gene encodes a protein consisting of five PDZ domains which serves as a scaffold to assemble different components of the phototransduction cascade, including the principal light-activated ion channels, the effector phospholipase C- $\beta$ and protein kinase C. Null inaD mutants have a dramatically reorganized subcellular distribution of signalling molecules, and a total loss of transduction complexes. Also, mutants defective in a single PDZ domain produce signalling complexes that lack the target protein and display corresponding defects in their physiology. A picture emerges of a highly organized unit of signalling, a 'transducisome', with PDZ domains functioning as key elements in the organization of transduction complexes in vivo.

The cellular responses to a wide variety of external signals are mediated by plasma-membrane receptors that transduce extracellular stimuli into an intracellular response. Although receptors that recognize different ligands are known to interact with the same intracellular signalling molecules, the specificity of signalling, which is often essential to a cell's physiological role, is maintained. But how is this specificity maintained, or rather, how is signal cross-talk avoided? One solution may be that different signalling cascades are organized into physically and functionally distinct signalling units. Response time, specificity and selectivity would then be greatly enhanced, and cross-talk would be minimized. Although we know much about the function and regulation of many signalling pathways, little is known about the architectural organization of the corresponding machinery.

Many of the molecules involved in signalling contain small protein-protein interaction domains that may allow recruitment and assembly into larger complexes. These include the Src-homology $\mathrm{SH} 2$ and $\mathrm{SH} 3$ domains ${ }^{1,2}$, pleckstrin-homology $(\mathrm{PH})^{3,4}$ and phosphotyrosine-binding $(\mathrm{PTB})^{5-7}$ domains, and postsynaptic density protein, disc-large, zo-1 (PDZ) domains ${ }^{8-12}$. Many of the proteins interacting with, or containing, PDZ domains are localized at the plasma membrane $e^{13-15}$, so PDZ domains may provide a framework for the recruitment of target molecules into membranebound macromolecular complexes. Recently, the PDZ-domain postsynaptic density protein PSD-95 has been shown to mediate the clustering of both NMDA ( $N$-methyl-D-aspartate) receptors ${ }^{12,16-18}$ and $\mathrm{K}^{+}$channels ${ }^{19-22}$. Furthermore, members of the PSD-95/93 family have been implicated in the clustering of synaptic complexes $^{16,23-25}$, and a PDZ domain in the protein tyrosinephosphatase Fap1 binds to the Fas membrane receptor ${ }^{26}$. Also, PDZ-PDZ interactions could be important mediators of proteinprotein interactions ${ }^{26}$. So far, PDZ domains have been found in more than 50 proteins, including many involved in cell signalling ${ }^{27-29}$, although little is known about the function of PDZ domains in vivo.

The Drosophila phototransduction cascade is a G-proteincoupled, phospholipase C (PLC) signalling pathway that shares many features with other signalling cascades ${ }^{30}$. Drosophila photoreceptor neurons also show a high degree of architectural organization, with most of the molecules involved in phototransduction localized to the rhabdomeres, a specialized subcellular compartment consisting of approximately 60,000 tightly packed microvilli, each $1-2 \mu \mathrm{m}$ in length and $50 \mathrm{~nm}$ wide ${ }^{31}$. In rhabdomeres, light activation of rhodopsin activates a $G_{q}$ protein $\alpha$-subunit $\left(G_{q \alpha}\right)$, which in turn activates PLC- $\beta^{32}$. PLC- $\beta$ catalyses the breakdown of phosphatidylinositol-4,5-bisphosphate $\left(\mathrm{PtdIns}_{2}\right)$ into the two intracellular messengers inositol trisphosphate $\left(\mathrm{InsP}_{3}\right)$ and diacylglycerol (DAG), leading to the eventual opening (and modulation) of the transient receptor potential (TRP) and TRPL light-activated channels ${ }^{33}$. Following termination of the stimulus, calcium-dependent regulatory processes, including activation of protein kinase $\mathrm{C}$ (eyePKC), mediate deactivation of the light response ${ }^{34}$. The inaD (inactivation no-after potential) locus is one of many loci identified in genetic screens designed to investigate this signalling cascade. A single mutant allele, inaD ${ }^{215}$, was isolated and shown to have a dominant-negative phenotype on photoreceptor deactivation ${ }^{35,36}$. Previous studies showed that the InaD protein contains at least two PDZ domains ${ }^{36}$ and interacts with the TRP ion channel ${ }^{37}$. InaD was also reported to associate with multiple components of the phototransduction cascade, including PLC- $\beta$, eye-PKC, rhodopsin and calmodulin $^{37-39}$. Thus it has been proposed that InaD is a regulatory component involved in feedback regulation of the light response $\mathrm{e}^{39}$. To determine the role of the PDZ-containing InaD protein in vivo, we sought to identify its interacting component(s), and to perform a genetic and physiological analysis of its function. We show that the InaD protein is composed of five distinct PDZ domains and acts as the organizing scaffold for photoreceptor signalling complexes in vivo. Our genetic, physiological and cell-biological studies demonstrate the presence of a multivalent adapter protein that links multiple signalling components within the same cascade. Our results also demonstrate that PDZ domains have a fundamental role in the assembly of transduction complexes in vivo.

\section{InaD protein consists of five PDZ domains}

InaD has been shown to associate with components of the phototransduction cascade ${ }^{37-39}$. Given the ability of PDZ domains to mediate protein-protein interactions, we sought to determine whether InaD is involved in the assembly of transduction complexes. We first defined the interaction between InaD and the 
interacting proteins, and then screened for Drosophila mutants defective in this molecule. When we analysed the primary structure of InaD in detail ${ }^{36}$, we found it to be a modular protein composed of five closely linked PDZ domains ${ }^{13}$ (Fig. 1). Each domain contains the structural characteristics of a prototypical PDZ motif, including the conservation of residues thought to be important for target binding ${ }^{27}$. However, the five domains display enough variability between them, and with other members of the PDZ domain family, to allow distinct protein-protein interactions ${ }^{40}$.

\section{Interaction with the phototransduction cascade}

Because InaD contains five distinct PDZ domains, we reasoned that it might interact with as many as five different components of the phototransduction cascade. We used immunoprecipitations and glutathione $S$-transferase (GST)-InaD fusion proteins to identify InaD targets. InaD antibodies co-immunoprecipitate TRP, eye-PKC and PLC- $\beta$ from head extracts ${ }^{38}$ (Fig. $2 a$ ), but not rhodopsin or $\mathrm{G}_{\mathrm{q} \alpha}$, despite the fact that both of these are extremely abundant in photoreceptor cells; identical results were obtained in vitro using a full-length GST-InaD fusion protein (Fig. 2b).

To identify the site on InaD that interacts with TRP, eye-PKC and PLC- $\beta$, we produced individual PDZ domains of InaD as GST fusion proteins and assayed them for interaction with each of these target proteins in whole head extracts (Fig. 2b). The third PDZ domain of InaD is specific for $\mathrm{TRP}^{37}$, the fourth domain interacts with eye-PKC, and the fifth domain interacts with PLC- $\beta$. Given the modular organization of PDZ domains in InaD, we next examined if binding to one target relies on the binding to the others. We found that immunoprecipitation of InaD from $\operatorname{trp}$ mutants, PLC- $\beta$ null mutants (norpA) or PKC null mutants (inaC) still co-precipitates the remaining two targets (Fig. 2a). These results demonstrate some important aspects of the function of InaD and PDZ domains: different PDZ domains have different and highly specific targets; InaD functions as a modular multivalent PDZ protein interacting with different components of the same pathway; and these interactions are not formed by interdependency between the different partners.

\section{inaD mutants do not form transduction complexes}

If InaD functions in vivo as a scaffold to localize or assemble components of the phototransduction cascade into transduction complexes, then a null inaD mutant should display a total loss of signalling complexes and a redistribution of individual signalling molecules. Unfortunately, only a single inaD mutant allele had been isolated and this behaved genetically and physiologically as a partly dominant-negative mutation ${ }^{35,36}$. We therefore tried to isolate new inaD alleles. We used a screening strategy based on the loss of InaD antigen on immunoblots, rather than on a hypothetical

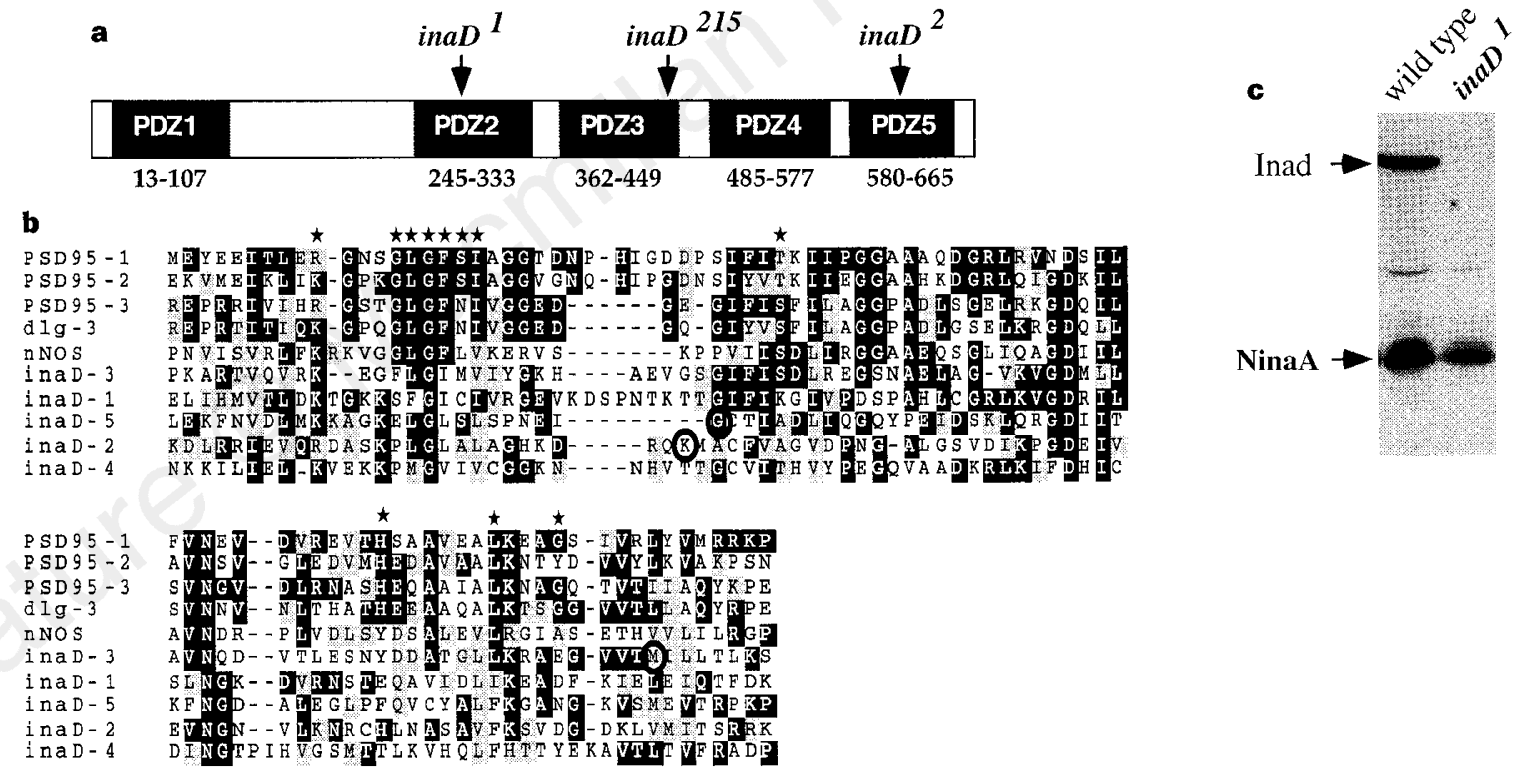

Figure 1 InaD is composed of five distinct PDZ domains. a, Diagram of the InaD protein, highlighting the location and size of each PDZ domain; also shown is the relative location of the three inaD mutations. $\mathbf{b}$, Amino-acid alignment of PDZ domains from mammalian PSD-95 (ref. 9), nNOS (ref. 23), Drosophila Dlg (ref. 8) and InaD (ref. 36). Sequences were aligned to maximize similarities. Black boxes indicate amino-acid identities, and grey boxes show conservative substitutions. Stars above the sequence indicate residues implicated in substrate binding ${ }^{27}$. The circles residues refer to the site of point mutation in the three Drosophila inaD alleles (see text for details). c, Immunoblot showing the absence of InaD protein in ina $D^{1}$. NinaA was used as a control.
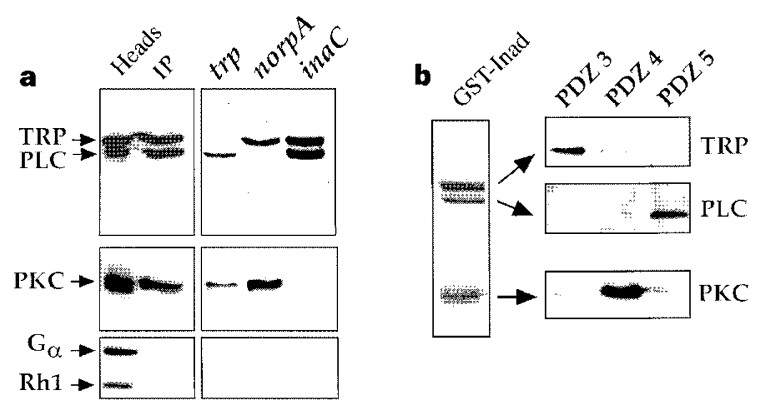

Figure 2 InaD forms a complex with PLC/PKC/TRP. a, Membranes prepared from the heads of wild-type flies (IP) or trp, norpA and inaC mutants were immunoprecipitated $(100 \mu \mathrm{g})$ with anti-InaD antibody. The immunoprecipitated proteins were separated by SDS-PAGE, transferred to nitrocellulose, and probed with antibodies specific for TRP, PLC, eye-PKC, and rhodopsin (Rh1). "Heads" refers to membranes before immunoprecipitation. InaD co-precipitates with TRP, PLC and PKC, but not with Rh1 or $G_{\alpha}$. As expected, immunoprecipitations from inaD nulls did not precipitate TRP, PKC or PLC (data not shown). b, A full-length GST-Inad fusion protein associates with TRP, PLC and PKC; overexpression of each of the individual PDZ domains from InaD as GST-PDZ fusions produce highly preferred interactions. 
physiological or behavioural basis. We generated fly stocks containing individual homozygous mutagenized second chromosomes (inaD maps to the second chromosome at position 591B1-2), and each stock was then subjected to immunoblot analysis for the loss of anti-InaD immunoreactivity ${ }^{33}$. Analysis of 2,847 lines yielded two alleles, inaD $D^{1}$ and $i n a D^{2}: i n a D^{1}$ has a complete loss of the protein, whereas inaD ${ }^{2}$ expresses reduced levels of protein.

By using the polymerase chain reaction (PCR), we isolated the inaD gene from each mutant allele and determined the entire nucleotide sequence. We found that $i n a D^{1}$ has an amber nonsense mutation at position 811 , leading to premature termination of the polypeptide chain at amino-acid residue 270; this represents a complete null allele. The inaD ${ }^{2}$ allele, however, has an A to $\mathrm{G}$ change at nucleotide 1814, leading to a substitution of a conserved glycine for glutamic acid in the fifth PDZ domain (Fig. 1).

We used immunofluorescent staining of frozen tissue sections to test for the subcellular localization of signalling molecules in the ina $D^{1}$ null mutant cells. TRP, PLC- $\beta$ and eye-PKC are completely mislocalized in the inaD $D^{1}$ mutant (Fig. 3). These proteins no longer localize to the rhabdomeres, but instead are found randomly distributed throughout either the plasma membrane (in the case of TRP) or the cytoplasm (PLC- $\beta$ and eye-PKC). In contrast, rhodopsin, $\mathrm{G}_{\mathrm{q} \alpha}$ and TRPL distribute normally in inaD ${ }^{1}$ (Fig. 3). Because signalling complexes are not formed in inaD $D^{1}$ mutants, photoreceptors have profound signalling defects, responding only at the highest light intensities and with dramatically altered kinetics (Fig. 4).

We suspected that a loss of the scaffold and mislocalization of the signalling proteins may result in the instability of these target proteins. We therefore examined the steady-state levels of transduction proteins by immunoblot analysis at different stages posteclosion. As predicted, the levels of TRP, PLC- $\beta$ and eye-PKC are all markedly reduced in the inaD $D^{1}$ mutants, and by 10 days posteclosion are less than $10 \%$ of wild-type levels (Fig. 5). In contrast, the levels of rhodopsin, $\mathrm{G}_{\mathrm{q} \alpha}$ and TRPL are unaffected. Taken together, these results demonstrate that InaD has an important role in organizing signalling complexes, and show that these complexes are required for the stability of the target proteins.

\section{Effects of mutations in PDZ domains}

We have shown that InaD interacts with multiple components of the phototransduction cascade and is essential for the assembly of signalling complexes. We reasoned that it should be possible to mutate the PDZ domain for a particular target and prevent the recruitment of that protein into the transduction complexes. This should generate in vivo phenotypes that resemble mutations in the target proteins.

The original inaD allele, inaD ${ }^{215}$, has a missense mutation in the third PDZ domain ${ }^{36}$. Because this domain is involved in the interaction of InaD with TRP (Fig. 2), and this mutation abolishes the interaction of TRP with $\mathrm{InaD}^{37,39}$, we sought to determine whether the stability of TRP, its subcellular localization and its function are disrupted in inaD ${ }^{215}$ mutants. We examined TRP protein levels by immunoblot analysis, its subcellular localization by immunofluorescent staining of tissue sections, and its function by performing whole-cell voltage-clamp recordings and electroretinograms.

We found that TRP protein levels decline with age in inaD ${ }^{215}$ mutants (Fig. 5). In young inaD ${ }^{215}$ flies (less than $24 \mathrm{~h}$ old), TRP levels are indistinguishable from control flies, but by 10 days the protein is barely detectable in immunoblots. In contrast, PLC- $\beta$, eye-PKC and other transduction protein levels remain constant. TRP channels are completely mislocalized in the inaD ${ }^{215}$ mutant (Fig. 6), and are found randomly distributed throughout the plasma membrane (we assayed newly eclosed flies to prevent degradation of TRP). To examine the mislocalization of TRP in more detail, we performed immuno-electronmicroscopic staining using goldconjugated secondary antibodies. These results (Fig. 6b) confirmed and extended the immunofluorescence observation that the TRP channel now localizes randomly to the plasma membrane. We found no evidence for mislocalization of any other phototransduction protein, including PLC- $\beta$ and eye-PKC (Fig. 6 and data not shown). Also, contrary to previous reports ${ }^{39}$, we never found TRP in the extracellular matrix, nor did we observe significant levels of cytoplasmic labelling.

To characterize the physiology of ina $D^{215}$ mutants we performed two sets of studies. Because immunoblot analysis revealed that levels of TRP protein decline with age in $i n a D^{215}$ mutants, we predicted that inaD $D^{215}$ cells should take on a TRP mutant phenotype over time. We found that inaD ${ }^{215}$ mutants display an electroretinogram (ERG) phenotype that approaches that of trp mutants (Fig. 7a), and does so on a time course similar to that of the decay of TRP protein seen in immunoblots (data not shown). We also performed wholecell voltage-clamp recordings of macroscopic currents and quantum
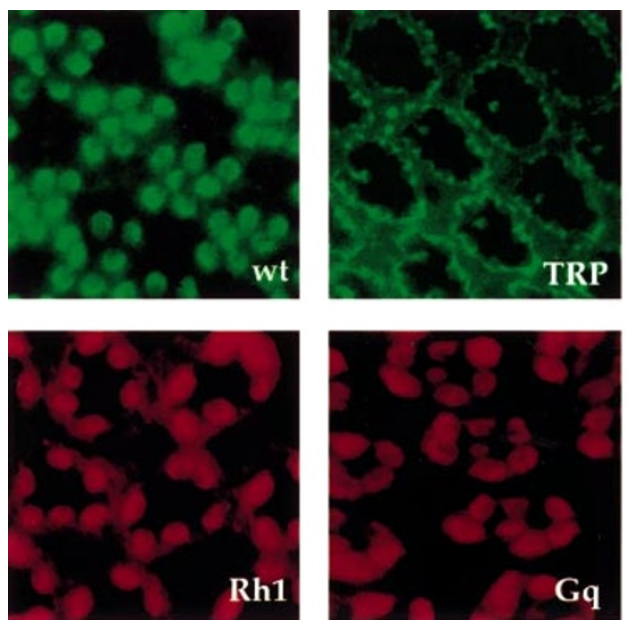

Figure 3 TRP, eye-PKC and PLC are mislocalized in ina $D^{1}$ null mutant photoreceptors. Immunofluorescent staining of cross-sections ( $1 \mu \mathrm{m}$ thick) of wild-type (wt, top left) and ina $D^{1}$ mutant (all other panels) photoreceptors for different transduction proteins. Top, ina $D^{1}$ mutant photoreceptors show exten-
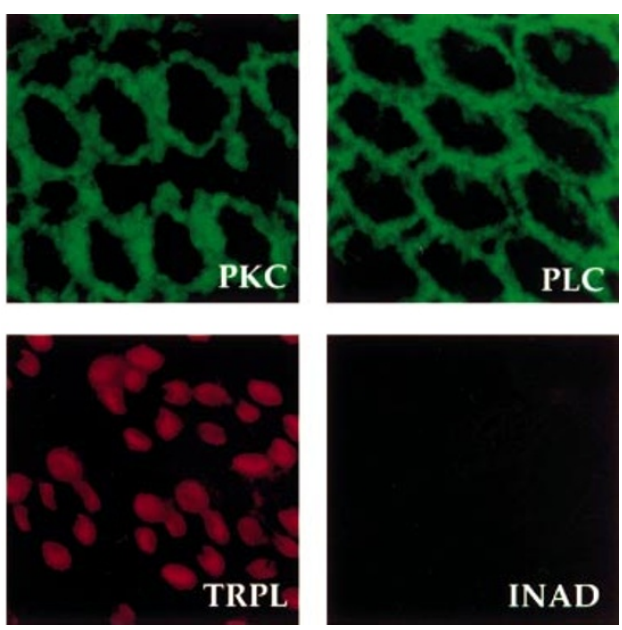

sive mislocalization of TRP, eye-PKC and PLC. Bottom, rhodopsin (Rh1), $\mathrm{G}_{\mathrm{q} \alpha}$ and TRP-like (TRPL) show normal rhabdomeric expression in ina $D^{1}$. Bottom right, the lack of Inad-immunoreactive material in the ina $D^{1}$ mutants. 
bumps. The ina $D^{215}$ mutants were originally characterized as displaying slow deactivation kinetics in response to a flash of light ${ }^{36}$ (Fig. 7b). This deactivation defect led others to postulate that InaD is involved in regulation of the phototransduction cascade, perhaps by modulating TRP function ${ }^{37-39}$. To determine the basis for the slow deactivation component in ina ${ }^{215}$ photoreceptors, we characterized quantal responses. In wild-type photoreceptors, single photons give rise to unitary events known as quantum bumps ${ }^{41,42}$. These quantum bumps are the result of the activation of a single rhodopsin molecule, and reflect the amplification of the entire signalling pathway, leading to the opening of the lightactivated channels ${ }^{43}$. Surprisingly, the quantum bumps from ina $D^{215}$ flies display normal termination kinetics (wild type, $t_{90 \%}=13.6 \pm 0.58 \mathrm{~ms} ;$ inaD ${ }^{215}, t_{90 \%}=13.8 \pm 0.60 \mathrm{~ms} ;$ Fig. $7 \mathrm{~b}$, right), indicating that the macroscopic defect of inaD ${ }^{215}$ mutants cannot be due to an underlying defect in deactivation. We reasoned that the apparent deactivation defect seen in the macroscopic response could be due to a bump activation defect. For instance, an increase in the latency of bump generation would be reflected as a broadly distributed macroscopic current ${ }^{44}$. Indeed, we found that the mean latency times between stimulus and quantum-bump generation were significantly altered in inaD ${ }^{215}$ mutants (wild type, $47.6 \pm 1.3 \mathrm{~ms}$; inaD ${ }^{215}, 67.0 \pm 3.2 \mathrm{~ms}$; Fig. $7 \mathrm{c}$ ). Thus inaD ${ }^{215}$ photoreceptors do not have a defect in termination ${ }^{36}$, nor do they display problems with feedback regulation ${ }^{37,39}$. Instead, the phenotype could be explained by understanding that the mislocalization of TRP channels leads to longer latencies and a corresponding macroscopic defect in deactivation kinetics.

To further define the physiological importance of the interaction

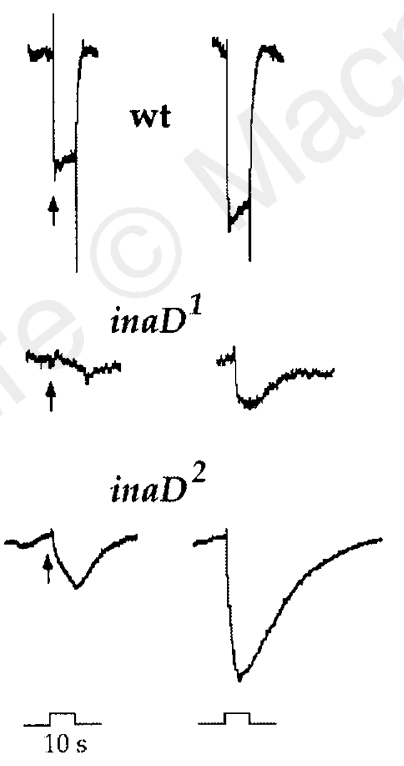

Figure 4 Photoresponses of $i n a D^{1}$ and $i n a D^{2}$. ERG recordings from wild type (wt), ina $D^{1}$ and ina $D^{2}$ mutant flies at $<1$ day after eclosion. The stimulus was a 10 -s pulse of orange light ( $570 \mathrm{~nm}$ longpass filter). Right traces show responses to 10 times the amount of light in the left traces $(\log [/]=-2$ and $\log [/]=-1$, respectively). Note the severe defects in the light responses from the mutant cells. Arrows indicate the onset of the stimulus. Both mutants have an increase in loss of responsiveness as a function of age. between InaD and its individual targets, we also analysed the interaction between InaD and PLC- $\beta$. Because ina $D^{2}$ has a mutation in the fifth PDZ domain (Fig. 1), and this domain is involved in the interaction with PLC- $\beta$ (Fig. 2), we sought to determine whether ina $D^{2}$ photoreceptors display a specific redistribution of PLC- $\beta$ in vivo. Indeed, PLC- $\beta$ is randomly distributed in the cytoplasm of ina $D^{2}$ photoreceptor cells (Fig. 6), whereas other transduction proteins, such as eye-PKC, TRP, Rh1 and $\mathrm{DG}_{\mathrm{q}}$, are unaffected. Consistent with its failure to be recruited into transduction complexes, PLC- $\beta$ becomes unstable and decays over time (Fig. 5).

Given the loss of PLC- $\beta$ from transduction complexes, we expected significant defects in phototransduction. ERG recordings from inaD $D^{2}$ mutant photoreceptors are shown in Fig. 4. These cells exhibit major defects in response kinetics: latency, activation and deactivation are all significantly slower in the mutant cells. Because these recordings were performed in newly eclosed flies, a time at which there are near normal levels of PLC (Fig. 5), they demonstrate that it is not the presence of a transduction molecule, but rather its location, that promotes effective signalling. Taken together, our data indicate the existence of a highly organized signalling unit, a transducisome, show that it is possible to manipulate experimentally the composition of signalling complexes, and demonstrate that PDZ domains play an essential role in the assembly and function of transduction complexes in vivo.

\section{Conclusions}

Cells can respond to a vast array of signals, and do so by activating the appropriate intracellular signalling pathways. Many different receptors share common downstream targets, but it has not always
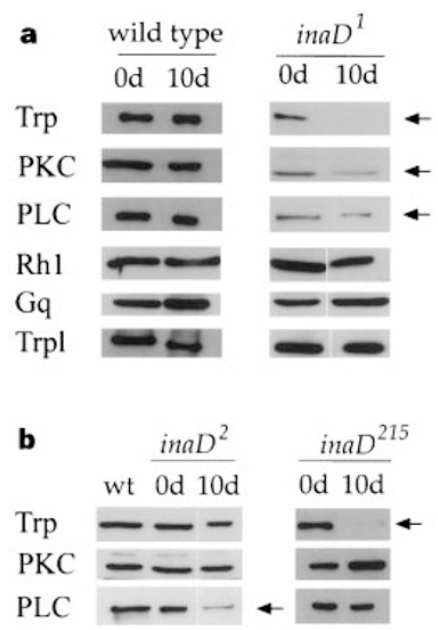

Figure $\mathbf{5}$ Transduction proteins that fail to associate with InaD become unstable. Immunoblot analysis of transduction proteins in wild type and inaD mutants. Protein levels at $<24 \mathrm{~h}$ after eclosion ( $0 \mathrm{~d}$ ) and after 10 days ( $10 \mathrm{~d}$ ) are shown. a, Levels of all transduction proteins in wild-type flies remained constant with age, whereas TRP, eye-PKC and PLC declined drastically in ina $D^{1}$ mutants (see arrows). b. Only TRP declines in ina ${ }^{215}$ (PDZ3), and only PLC declines in inaD ${ }^{2}$ (PDZ5) (see arrows). The equivalent of one fly head per lane was run for wild type and ina $D^{215}$, but two fly heads for ina $D^{1}$ and ina $D^{2}$. 
been clear how different pathways maintain specificity. Several lines of evidence suggest that signalling events do not occur freely in the cytosol of the cell, but rather are organized as architecturally and spatially distinct ultra-microdomains of signalling. In this way, a cell can optimize and tune its responses to different pathways by controlling the recruitment of different signalling molecules into the different transduction complexes, thereby enhancing specificity and speed yet minimizing cross-talk. An example is the yeast mating response, in which the product of the sterile 5 gene functions as a scaffold protein, coordinating the recruitment of several kinases within the same signalling pathway ${ }^{45-47}$.

We have used phototransduction in Drosophila as a model to study the organization of G-protein-coupled transduction complexes in vivo. In this signalling pathway, photoreceptor neurons report activity with remarkable sensitivity and specificity. Furthermore, photoreceptors achieve excellent temporal resolution by ensuring that the transduction machinery is reset quickly after generating a response. Phototransduction in Drosophila is the fastest known G-protein-coupled cascade, taking just a few tens of milliseconds to go from light activation of rhodopsin to the generation of a receptor potential, and less than $100 \mathrm{~ms}$ to shut off after termination of the stimulus ${ }^{30}$. InaD coordinates the recruitment of components involved in both activation (PLC- $\beta$ and TRP) and deactivation (eye-PKC) ${ }^{34}$. Our results suggest that an important strategy used by photoreceptors to attain high response speed is the assembly of signalling molecules into organized transducisomes. Therefore response time would not be limited by the diffusion of different signalling components within a microvillus.

A photoreceptor neuron attains high sensitivity by having an exceptionally large number of receptor molecules in its surface $\left(\sim 100 \times 10^{6} \text { rhodopsins per cell }\right)^{48,49}$. To couple this large number of receptor molecules to the downstream transduction complexes rapidly and efficiently would require a diffusable coupling molecule, in this case a $G$ protein. In this model, each $G$ protein would need to sample only a small number of receptors in the membrane (an ultramicrodomain of signalling) and report their activity to the downstream transduction complexes. Our finding that neither rhodopsin or $\mathrm{G}_{\alpha}$ are included in the InaD complexes supports this model. What do these complexes tell us about unitary (single-photon) responses? A quantum bump represents the coordinated activation of a few hundred light-activated ion channels in response to the activation of a single rhodopsin molecule ${ }^{30}$. The organization of InaD complexes into a supramolecular complex, either through PDZ-PDZ domain interactions or PDZ-cytoskeletal interactions within a microvillus, could represent the structural basis of a quantum bump, ensuring both reliability and coordinated signalling. Our observation that PLC is positioned in close proximity to the light-activated channels through $\mathrm{InaD}$ provides an intriguing possibility for a direct gating mechanism. Also, the finding that the TRPL channels remain properly localized in inaD mutants further supports the postulate that TRP and TRPL are not likely to form or function as heteromultimers in vivo ${ }^{33}$.

The structural basis of the interaction between PDZ domains and their targets is becoming clear. For instance, the X-ray crystallographic structure of the third PDZ domain from the synaptic protein PSD-95, alone or in complex with its peptide ligand, has recently been determined ${ }^{5,27,28}$. In addition, binding studies with oriented peptide libraries showed that different PDZ domains a
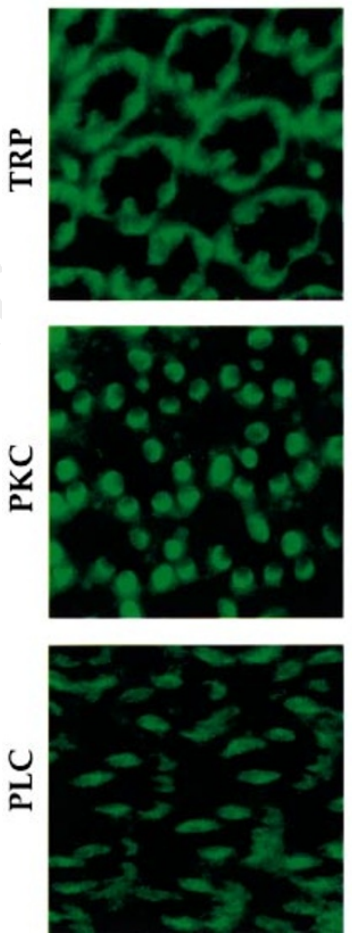

ina $D^{2}$
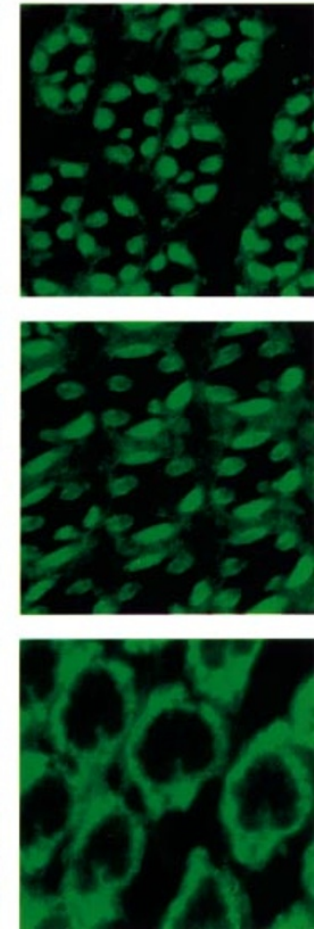

b
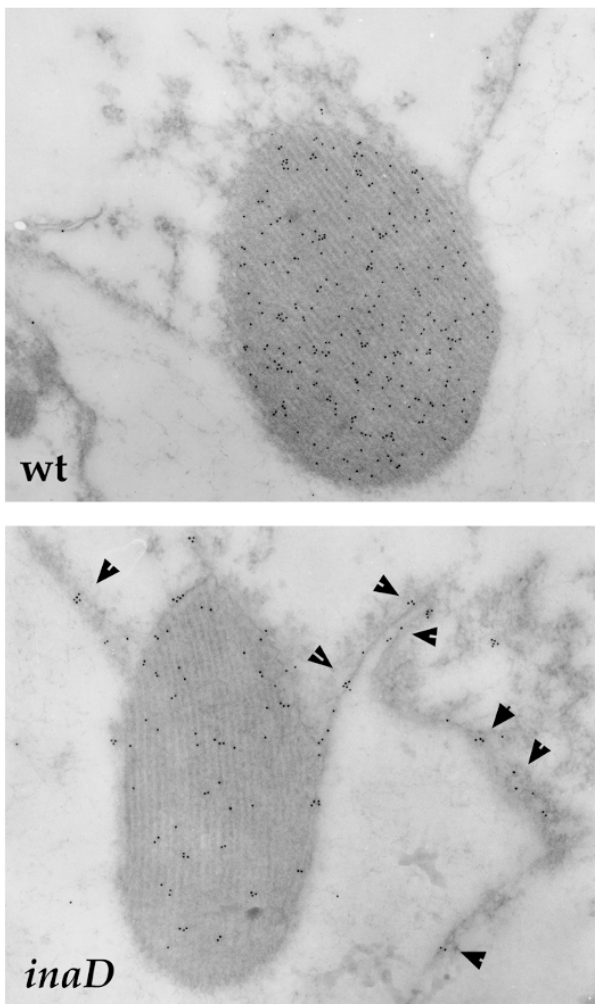

Figure 6 ina $D^{215}$ and $i n a D^{2}$ mutants show specific mislocalization of TRP and PLC. a, Immunofluorescent staining for TRP, eye-PKC and PLC (as indicated) in cross-section ( $1 \mu \mathrm{m}$ thick) of ina $D^{215}$ (left) and ina $D^{2}$ (right) mutant photoreceptors. TRP is mislocalized in ina $D^{215}$ mutants, whereas PLC is mislocalized in ina $D^{2}$ mutants. All other transduction proteins show normal rhabdomeric localization in either genetic background. b. Electronmicroscopic immunogold localization of
TRP in wild-type (wt) and ina $D^{215}$ rhabdomeres ${ }^{31,33}$. In wild-type photoreceptors, TRP is present at significantly higher levels and exclusively in the microvillar membranes of the rhabdomeres; in ina $D^{215}$, TRP levels are significantly reduced in the rhabdomeres (newly eclosed flies), and is distributed prominently throughout the plasma membrane (arrows). 
display preferences for distinct targets ${ }^{40}$. These in vitro studies suggested that, although all PDZ domains may share structural elements, different PDZ domains should show specificity for different target proteins. We have now shown this to be the case in vivo. The finding that $\mathrm{InaD}$ is composed primarily of $\mathrm{PDZ}$ domains strengthens the importance of this protein motif in the organization of signalling pathways. Recently, GRIP (glutamate receptor interacting protein), a new protein composed solely of seven PDZ domains, has been identified in mammalian cells ${ }^{50}$. It has not yet been determined whether this molecule also functions as a

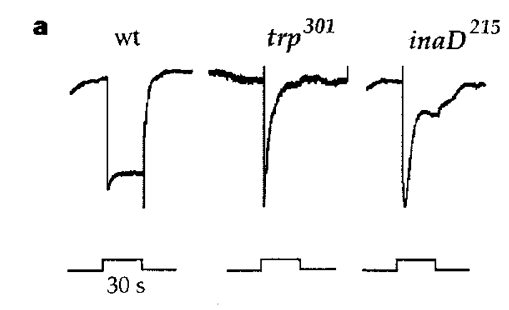

b
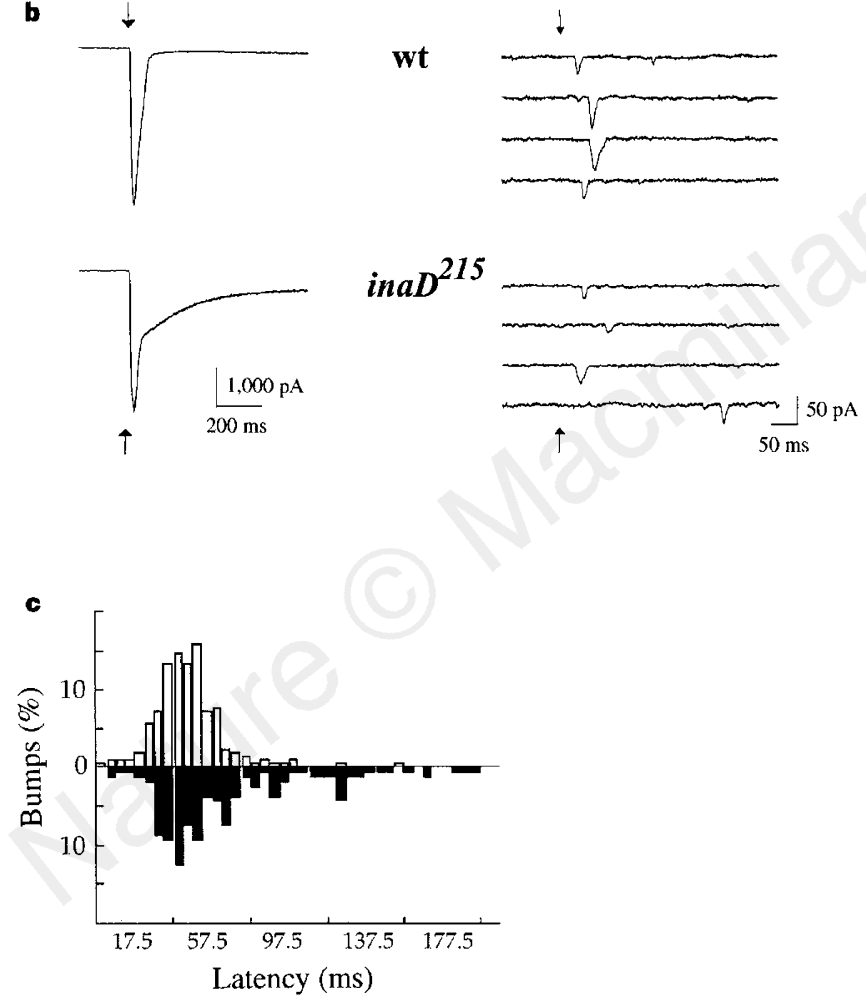

Figure 7 Photoresponses of ina $D^{215}$. a, ERG recordings from wild-type (wt), trp ${ }^{301}$ and ina $D^{215}$ mutant eyes at 20 days after eclosion. Light stimulus was a 30 -s pulse of orange light $(570 \mathrm{~nm}$ longpass filter). Note the transient response of trp (transient receptor potential) mutants and older ina $D^{215}$ flies. b. Whole-cell recordings (left) and quantum bumps (right) from wild-type and ina $D^{215}$ mutant photoreceptors. For macroscopic responses, cells were stimulated (arrow) with a $10-\mathrm{ms}$ flash of $580 \mathrm{~nm}$ light of $\log [/]=1$. The deactivation time course of $i n a D^{215}$ is well fitted by the sum of two exponentials (time constants of $14.7 \pm 2.5$ and $143.1 \pm 12.1 \mathrm{~ms} ; N=7$ ), whereas the time course of decay of wild-type responses is fitted by a single exponential with a time constant of $14.5 \pm 2.2 \mathrm{~ms}(N=6)$. For quantum bumps ${ }^{44}$, cells were stimulated with a 10-ms flash of $580 \mathrm{~nm}$ light of $\log [/]=-6.5$ (arrow). This stimulus produced a probability of not seeing a bump of 0.40 in wild type and 0.65 in ina $D^{215}$. Note the normal termination kinetics of ina $D^{215}$ bumps. c, ina $D^{215}$ quantum bumps have defective latency. Latency to first bump from wild type (open bars) and ina $D^{215}$ mutant (solid bars) were $47.6 \pm 1.3 \mathrm{~ms}(N=210$ bumps from 7 cells), and $67.0 \pm 3.1 \mathrm{~ms}(N=160$ bumps from 7 cells), respectively. signalling scaffold, organizing specific transduction complexes at the synapse. Current models of PDZ-target interaction involve a binding site composed of S/TXV residues at the carboxy-terminal end of the target protein. All three InaD targets lack such a motif (position -4 from the $\mathrm{C}$ terminus in TRP, positions -122 and -507 in eye-PKC, and several sites PLC- $\beta$, the closest at residue -26), which argues against an exclusive model requiring that PDZ domains bind the absolute C-terminal end of their target proteins ${ }^{5,27,29,40}$. We also showed that InaD functions as a modular protein, and demonstrated that eliminating one target does not prevent InaD from interacting with the others. The identification of all of the InaD targets and the corresponding PDZ sites should provide an experimentally tractable system in which to define the structural basis of PDZ-target interaction in vivo. It may even be possible to custom-design transduction complexes by manipulating the number, orientation and distribution of PDZ domains in InaD. The availability of a null inaD mutant background now makes these studies possible.

\section{Methods}

Mutant screen and western blots. Males of Drosophila $c n$ bw genotype were aged for 5 days, treated with EMS, and crossed to flies carrying the dominant temperature-sensitive DTS91 allele. Single $\mathrm{F}_{1}$ males were collected and crossed in single vials to CyO/DTS91 virgin females. The vials were then shifted to $29^{\circ} \mathrm{C}$ for $72 \mathrm{~h}$ to eliminate any eggs or larvae carrying the DTS allele. The parents were then removed and the vials were incubated at $29^{\circ} \mathrm{C}$ for an additional $48 \mathrm{~h}$ before being returned to $25^{\circ} \mathrm{C}$. The progeny from this cross were transferred to fresh food, and their homozygous white-eyed offspring ( $c n b w)$ were subjected to a protein immunoblot screen for the loss of the InaD antigen ${ }^{33}$.

Antibodies. To generate antibodies specific to InaD, we generated a T7 fusion protein consisting of the last 300 residues of the protein. All antibodies were checked for specificity and affinity using wild-type, mutant and transgenic controls. For immunostaining, the InaD antibody was diluted 1:500 in phosphate buffered saline (PBS), 1\% BSA, 0.1\% saponin (PBS-S); the TRP antibody was first preabsorbed with a homogenate of trp mutant heads to reduce background staining and used at a final dilution of $1: 100$. Rhodopsin ( $1: 300)$, eye-PKC $(1: 50)$, PLC $(1: 1000)$, TRPL $(1: 100)$ and DG $_{\mathrm{q}}(1: 200)$ were detected using polyclonal antibodies ${ }^{33,34,44}$

Immunoprecipitation. Frozen heads (500-1,000) were homogenized in 2$3 \mathrm{ml}$ of buffer A (50 mM Tris- $\mathrm{HCl}, \mathrm{pH} 7.5,50 \mathrm{mM} \mathrm{NaCl}, 1 \mathrm{mM}$ EDTA and protease inhibitors) using a glass-glass homogenizer. The homogenate was centrifuged at 4,000 $\mathrm{g}$ for $1 \mathrm{~min}$ to remove chitinous material. Membranes were isolated by centrifugation at $100,000 \mathrm{~g}$ for $30 \mathrm{~min}$ at $4{ }^{\circ} \mathrm{C}$, and resuspended in $0.8-1.0 \mathrm{ml}$ of buffer A to determine protein concentration. Samples were recentrifuged, resuspended in buffer $\mathrm{B}(150 \mathrm{mM} \mathrm{NaCl}, 1 \%$ triton, $50 \mathrm{mM}$ Tris$\mathrm{HCl}, \mathrm{pH} 8.0$, and protease inhibitors), mixed ( $100 \mu \mathrm{g}$ of protein) with antiInaD antibodies, and incubated for $1 \mathrm{~h}$ at $4{ }^{\circ} \mathrm{C}$. At this time, $30 \mu \mathrm{l}$ of protein A-agarose beads (Pierce) were added and incubated for $2 \mathrm{~h}$. Samples were washed in buffer B, resuspended in SDS buffer and fractionated by SDS-PAGE. The entire immunoprecipitate was loaded on the gels. Studies using GST-InaD protein fusions used similar incubation conditions but also contained affinitypurified GST fusion proteins. GST fusions containing individual PDZ domains (PDZ1 to PDZ5) were constructed according to the boundaries shown in Fig. 1. All GST fusion proteins were overproduced and purified by affinity chromatography on glutathione-agarose beads ${ }^{19,23,50}$.

Electrophysiological recordings. Photoreceptors were isolated from adult flies ( $<6 \mathrm{~h}$ after eclosion) and whole-cell, patch-clamp recordings were performed $^{33}$. Photoreceptors were stimulated by a 75-W Xenon source connected to the epifluorescence port of an inverted Fluovert FS (Leitz) microscope; light was bandpass-filtered $(\lambda=580 \pm 10 \mathrm{~nm})$ and focused onto the photoreceptor cells with a 0.5 numerical aperture, $40 \times$ objective. Signals were recorded with an Axopatch 200A patch-clamp amplifier (Axon Instruments, Foster City, CA), and data were analysed using PCLAMP6.02 (Axon) and ORIGIN (Microcal) software. The membrane of the photoreceptors was voltage-clamped at a holding potential of $-40 \mathrm{mV}$. Traces were low-pass filtered at $2 \mathrm{kHz}$ (Bessel filter) and digitized at $2 \mathrm{kHz}$, unless stated otherwise. Measured series resistance, $16 \mathrm{M} \Omega$ on average, was $80 \%$ compensated. The bath solution 
contained (in $\mathrm{mM}$ ): $124 \mathrm{NaCl}, 4 \mathrm{KCl}, 10$ HEPES, 5 proline, 25 sucrose, $1.5 \mathrm{CaCl}_{2}, 1 \mathrm{MgCl}_{2}, \mathrm{pH}$ 7.15. Pipette solution contained $95 \mathrm{~K}$-gluconate, $40 \mathrm{KCl}, 10 \mathrm{HEPES}, 2 \mathrm{MgCl}_{2}, 2 \mathrm{EGTA}, \mathrm{pH}$ 7.15. For quantum-bump analysis, photoreceptors were clamped at $-70 \mathrm{mV}$, and stimulated with a dim light flash to generate quantum bumps around $50 \%$ of the time. Signals were lowpassfiltered at $1 \mathrm{kHz}$ and digitized at $2 \mathrm{kHz}$.

Received 11 April; accepted 13 June 1997.

1. Schlessinger, J. SH2/SH3 signaling proteins. Curr. Opin. Genet. Dev 4, 25-30 (1994).

2. Pawson, T. SH2 and SH3 domains in signal transduction. Adv. Cancer Res. 64, 87-110 (1994)

3. Lemmon, M. A., Ferguson, K. M. \& Schlessinger, J. PH domains: diverse sequences with a common fold recruit signaling molecules to the cell surface. Cell 85, 621-624 (1996).

4. Shaw, G. The plackstrin homology domain: an intriguing multifunctional protein module. Bioessays 18, 35-46 (1996).

5. Harrison, S. C. Peptide-surface association: the case of PDZ and PTB domains. Cell 86, 341-343 (1996).

6. van der Greer, P. \& Pawson, T. The PTB domain: a new protein module implicated in signal transduction. Trends Biochem. Sci. 20, 277-280 (1995).

7. Kavanaugh, W. M., Turck, C. W. \& Williams, L. T. PTB domain binding to signaling proteins through a sequence motif containing phosphotyrosine. Science 268, 1177-1179 (1995).

8. Woods, D. F. \& Bryant, P. J. The discs-large tumor suppressor gene of Drosophila encodes a guanylate kinase homolog localized at septate junctions. Cell 66, 451-464 (1991).

9. Cho, K. O., Hunt, C. A. \& Kennedy, M. B. The rat brain postsynaptic density fraction contains homolog of the Drosophila discs-large tumor suppressor protein. Neuron 9, 929-942 (1992).

10. Woods, D. F. \& Bryant, P. J. ZO-1, DlgA and PSD-95/SAP90: homologous proteins in tight, septate and synaptic cell junctions. Mech. Dev. 44, 85-89 (1993).

11. Kennedy, M. B. Origin of PDZ (DHR, GLGF) domains. Trends Biochem. Sci. 20, 350 (1995).

12. Kornau, H. C., Schenker, L. T., Kennedy, M. B. \& Seeburg, P. H. Domain interaction between NMDA receptor subunits and the postsynaptic density protein PSD-95. Science 269, 1737-1740 (1995).

13. Saras, J. \& Heldin, C. H. PDZ domains bind carboxy-terminal sequences of target proteins. Trends Biochem. Sci. 21, 455-458 (1996).

14. Sheng, M. \& Kim, E. Ion channel associated proteins. Curr. Opin. Neurobiol. 6, 602-608 (1996).

15. Sheng, M. PDZs and receptor/channel clustering: rouding up the latest suspects. Neuron 17, 575-578 (1996).

16. Muller, B. M. et al. SAP102, a novel postsynaptic protein that interacts with NMDA receptor complexes in vivo. Neuron 17, 255-265 (1996).

17. Niethammer, M., Kim, E. \& Sheng, M. Interaction between the C terminus of NMDA recepto subunits and multiple members of the PSD-95 family of membrane-associated guanylate kinases. J. Neurosci. 16, 2157-2163 (1996).

18. Kim, E., Cho, K. O., Rothschild, A. \& Sheng, M. Heteromultimerization and NMDA receptorclustering activity of Chapsyn-110, a member of the PSD-95 family of proteins. Neuron 17, 103-113 (1996).

19. Kim, E., Niethammer, M., Rothschild, A., Jan, Y. N. \& Sheng, M. Clustering of Shaker-type K ${ }^{+}$channels by interaction with a family of membrane-associated guanylate kinases. Nature $378,85-88$ (1995).

20. Kim, E. \& Sheng, M. Differential $\mathrm{K}^{+}$channel clustering activity of PSD-95 and SAP97, two related membrane-associated putative guanylate kinases. Neuropharmacology 35, 993-1000 (1996).

21. Cohen, N. A., Brenman, J. E., Snyder, S. H. \& Bredt, D. S. Binding of the inward rectifier K ${ }^{+}$channel Kir 2.3 to PSD-95 is regulated by protein kinase A phosphorylation. Neuron 17, 759-767 (1996).

22. Kim, E. et al. GKAP, a novel synaptic protein that interacts with the guanylate kinase-like domain of the PSD-95/SAP90 family of channel clustering molecules. J. Cell Biol. 136, 669-678 (1997).

23. Brenman, J. E. et al. Interaction of nitric oxide synthase with the postsynaptic density protein PSD-95 and $\alpha$-syntrophin mediated by PDZ-domains. Cell 84, 757-767 (1996).

24. Budnik, V. et al. Regulation of synapse structure and function by the Drosophila tumor suppressor gene dlg. Neuron 17, 627-640 (1996).

25. Brenman, J. E., Christopherson, K. S., Craven, S. E., McGee, A. W. \& Brdt, D. S. Cloning and characterization of postsynaptic density 93 , a nitric oxide synthase interacting protein. J. Neurosci. 16, 7407-7415 (1996).
26. Sato, T., Irie, S., Kitada, S. \& Reed, J. C. FAP-1: a protein tyrosine phosphatase that associates with Fas. Science 268, 411-415 (1995).

27. Doyle, D. A. et al. Crystal structures of a complexed and peptide-free membrane protein-binding domain: molecular basis of peptide recognition by PDZ. Cell 85, 1067-1076 (1996).

28. Cabral, J. H. et al. Crystal structure of a PDZ domain. Nature 382, 649-652 (1996).

29. Fanning, A. S. \& Anderson, J. M. Protein-protein interactions: PDZ domain networks. Curr. Biol. 6, 1385-1388 (1996).

30. Ranganathan, R., Malicki, D. M. \& Zuker, C. S. Signal transduction in Drosophila photorctprs. Annu. Rev. Neurosci. 18, 283-317 (1995).

31. Suzuki, E., Katayama, E. \& Hirosawa, K. Structure of photoreceptive membranes of Drosophila compound eyes as studies by quick-freezing electron microscopy. J. Electron Microsc. 42, 178-184 (1993)

32. Pak, W. L. Drosophila in vision research. The Friedenwald Lecture. Invest. Ophthalmol. Vis. Sci. 36, 2340-2357 (1995).

33. Niemeyer, B. A., Suzuki, E., Scott, K., Jalink, K. \& Zuker, C. S. The Drosophila light-activated conductance is composed of the two channels TRP and TRPL. Cell 85, 651-659 (1996).

34. Smith, D. P. et al. Photoreceptor deactivation and retinal degeneration mediated by a photoreceptorspecific protein kinase C. Science 254, 1478-1484 (1991).

35. Pak, W. L. Mutants Affecting the Vision in Drosophila melanogaster (Plenum, New York, 1975).

36. Shieh, B.-H. \& Niemeyer, B. A novel protein encoded by the InaD gene regulates recovery of visual transduction in Drosophila. Neuron 14, 201-210 (1995).

37. Shieh, B. H. \& Zhu, M. Y. Regulation ofthe TRP $\mathrm{Ca}^{2+}$ channel by INAD in Drosophila photoreceptors. Neuron 16, 991-998 (1996).

38. Huber, A. et al. The transient receptor potential protein $(\operatorname{Trp})$, a putative store-operated $\mathrm{Ca}^{2+}$ channel essential for phosphoinositide-mediated photoreception, forms a signaling complex with NorpA, InaC and InaD. EMBO J. 15, 7036-7045 (1996).

39. Chevesich, J., Kreuz, A. J. \& Montell, C. Requirement for the PDZ domain protein, INAD, for localization of the TRP store-operated channel to a signaling complex. Neuron 18, 95-105 (1997).

40. Songyang, Z. et al. Recognition of unique carboxyl-terminal motifs by distinct PDZ domains. Science 275, 73-77 (1997).

41. Yeandle, S. thesis, Johns Hopkins Univ. (1957).

42. Baylor, D. A., Lamb, T. D. \& Yau, K.-W. Responses of retinal rods to single photons. J. Physiol. (Lond.) 288, 613-634 (1979).

43. Minke, B. Is the receptor potential of fly photoreceptors a summation of single-photon responses? Comments Theor. Biol. 3, 229-263 (1994).

44. Scott, K., Leslie, A., Sun, Y., Hardy, R. \& Zuker, C. G $\alpha$ q protein function in vivo: Genetic dissection of its role in photoreceptor cell physiology. Neuron 15, 919-927 (1995).

45. Marcus, S., Polverino, A., Barr, M. \& Wigler, M. Complexes between STE5 and components of the pheromone-responsive mitogen-activated protein kinase module. Proc. Natl Acad. Sci. USA 91, 77627766 (1994)

46. Printen, J. A. \& Sprague, G. J. Protein-protein interactions in the yeast pheromone response pathway: Ste5p interacts with all members of the MAP kinase cascade. Genetics 138, 609-619 (1994).

47. Choi, K. Y., Satterberg, B., Lyons, D. M. \& Elion, E. A. Ste5 tethers multiple protein kinases in the MAP kinase cascade required for mating in S. cerevisiae. Cell 78, 499-512 (1994).

48. Larrivee, D. C., Conrad, S. K., Stephenson, R. S. \& Pak, W. L. Mutation that selectively affects rhodopsin concentration in the peripheral photoreceptors of Drosophila melanogaster. J. Gen. Physiol. 78, 521-545 (1981)

49. Johnson, E. \& Pak, W. Electrophysiological study of Drosophila rhodopsin mutants. J. Gen. Physiol. 88, 651-673 (1986).

50. Hualing, D. et al. GRIP: a synaptic PDZ domain-containing protein that interacts with AMPA receptors. Nature 386, 279-284 (1997).

Acknowledgements. We thank B. Niemeyer for advice, initial observations and discussions; D. Schultz for help with the yeast two-hybrid system; E. Koundakjian and D. Cowan for help with the $\mathrm{F}_{3}$ screen; and R. MacKinnon and members of the Zuker laboratory for discussions. S.T. is a fellow of the American by a Markey predoctoral training grant; and C.S.Z. is an investigator of the Howard Hughes Medical Institute.

Correspondence and requests for materials should be addressed to C.S.Z. (e-mail: czuker@ucsd.edu) 